\title{
PRÁTICAS ALIMENTARES E SUA RELAÇÃO COM AS INTERCORRÊNCIAS CLÍNICAS DE CRIANÇAS DE ZERO A SEIS MESES
}

\author{
Alimentary practices and their relation with clinical \\ intercurrences of children from zero to six months age \\ Prácticas alimenticias y su relación con las intercurrencias \\ clínicas de niños de cero a seis meses
}

\author{
Lorena Barbosa Ximenes ${ }^{1}$ \\ Juliane Girão de Moura ${ }^{2}$ Mônica Oliveira Batista Oriá ${ }^{3}$ \\ Mariana Cavalcante Martins ${ }^{4}$ \\ Paulo Cesar de Almeida ${ }^{5}$ \\ Elioneide Paulo Carneiro ${ }^{6}$
}

\section{RESUMO}

Este estudo foi desenvolvido para identificar os principais agravos de saúde em crianças de zero a seis meses de vida e verificar a relação entre os agravos de saúde e as práticas alimentares mais frequentes. Foram analisadas informações de 36 crianças, identificadas primeiramente na faixa etária de zero a dois meses e acompanhadas até seis meses em uma Unidade de Cuidado de Enfermagem do Centro de Desenvolvimento Familiar (CEDEFAM) em 134 encontros, por meio de formulário estruturado. Houve predomínio de $24(18 \%)$ crianças na faixa etária entre um e dois meses, sendo que $15(11,2)$ dessas se encontravam em AME (aleitamento materno exclusivo). Quanto aos agravos relacionados aos lactentes em AME, houve 75 ocorrências (40,5\%), sendo $33(17,8 \%)$ problemas respiratórios, 38 (20,5\%) problemas dermatológicos e 4 (2,2\%) problemas gastrointestinais. Portanto, as crianças, principalmente as menores de seis meses de idade, fazem parte de um grupo mais vulnerável, e o leite materno pode reduzir a morbimortalidade infantil.

Palavras-chave: Hábitos Alimentares. Saúde da Criança. Cuidados de Enfermagem.

\begin{abstract}
This study was developed to identify the main health damages, as well as practices of the more frequent feeding among children from zero to six months of life. It was analyzed the data from 36 children, identifying firstly from the age of 0 to 2 months and accompanied until six months in a Nursing Care Unit of the Center for Family Development (CEDEFAM) in 134 meetings, using a structured form. There was a predominance of $24(18 \%)$ children in the age 1 and 2 months, and 15 (11.2) from these were in exclusive breastfeeding (EB). Among damages related to infants in $E B$, there were 75 occurrences (40.5\%), being 33 (17.8\%) respiratory problems, $38(20,5 \%)$ dermatological problems and $4(2.2 \%)$ gastrointestinal problems. Thus, the children, especially fewer than six months old, are part of a more vulnerable group, where the maternal milk can reduce the infant morbimortality.
\end{abstract}

Keywords: Food Habits. Child Health. Nursing Care.

\section{Resumen}

Este estudio fue desarrollado para identificar los principales agravios de salud en niños de cero a seis meses y verificar la relación entre los agravios de salud y las prácticas alimenticias más frecuentes. Fueron analizadas informaciones de 36 niños, identificadas primeramente en la faja de edad de 0 a 2 meses y acompañadas hasta seis meses en una Unidad de Atención de Enfermería del Centro de Desarrollo Familiar (CEDEFAM) en 134 encuentros, a través de encuestas estructuradas. Hubo predominio de 24 (18\%) niños en la faja de edad entre uno y dos meses de vida, siendo que $15(11,2)$ se encontraban en amamantamiento materno exclusivo (AME). Entre los agravios relacionados a los lactantes en AME, hubo 75 ocurrencias $(40,5 \%)$, siendo $33(17,8 \%)$ problemas respiratorios, 38 $(20,5 \%)$ problemas dermatológicos y $4(2,2 \%)$ problemas gastrointestinales. Así, los niños, principalmente, los menores de seis meses de edad, forman parte de un grupo más vulnerable, cuya la leche materna puede reducir la morbimor talidad infantil.

Palabras clave: Hábitos Alimenticios. Salud del Niño. Atención de Enfermería.

'Doutora em Enfermagem. Docente Adjunta IV do Departamento de Enfermagem da Universidade Federal do Ceará - UFC. Bolsista Produtividade do CNPq, Nível 2.Brasil E-mail: Ibximenes@yahoo.com,²Enfermeira da Triagem Clínica do Centro de Hematologia e Hemoterapia do Ceará - Hemoce. Discente da Especialização em Enfermagem Oncológica. Brasil. E-mail: julianemoura@hotmail.com, ${ }^{3}$ Doutora em Enfermagem. Post Doc (University of Virginia, USA). Docente Adjunta I do Departamento de Enfermagem da Universidade Federal do Ceará - UFC. Brasil. E-mail: oriaremon@hotmail.com, ${ }^{4}$ Mestre em Saúde Coletiva. Discente do curso de Doutorado em Enfermagem da Universidade Federal do Ceará - UFC. Bolsista da CAPES. Brasil. E-mail: marianaenfermagem@hotmail.com, ${ }^{5}$ Doutor em Saúde Pública. Docente da Universidade Estadual do Ceará. Brasil. E-mail: pc_almeida@zipmail.com.br, ${ }^{6}$ Especialista em Enfermagem Obstétrica. Enfermeira Supervisora de Estágio na área de Saúde Comunitária do Departamento de Enfermagem da UFC. Brasil. E-mail: elioneidepc@hotmail.com 


\section{Práticas alimentares e intercorrências clínicas}

\section{INTRODUCÃO}

0 leite materno, aquisição de matéria-prima essencial e de fonte de energia no início da vida, é imprescindível tanto para o crescimento como para o desenvolvimento físico e psicológico do ser humano. 0 ato de amamentar é de enorme importância por favorecer o contato materno, tendo efeito tranquilizante e analgésico sobre o lactente.

A amamentação e a interação materno-infantil são fatores que podem modular o estado comportamental da criança e da mãe, influenciando o desenvolvimento psicológico, tanto afetivo como de aprendizado. ${ }^{1}$

Uma das causas apontadas para o desmame precoce, por exemplo, é o desconhecimento da mãe em relação à qualidade de seu leite, não somente para sanar a fome, mas também para conduzir a um favorável desenvolvimento do seu bebê. Outros fatores que também contribuem para isso são as condições culturais e as relações pessoais.?

Crianças que estão em aleitamento materno exclusivo ao seio sofrem menos com agravos como diarreia, desidratação e risco de hospitalização por pneumonia e apresentam ganhos na área cognitiva e proteção contra doença atópica. ${ }^{3}$ Além disso, os lactentes que são amamentados exclusivamente por seis meses crescem com menos riscos de infecção do trato gastrintestinal, infecções respiratórias agudas e alergias. ${ }^{4-5}$

A cada ano, cerca de $55 \%$ das mortes de lactentes ocorrem em razão de doenças diarreicas e infecções respiratórias agudas que resultam de práticas errôneas de alimentação. Menos de 35\% dos lactentes de todo o mundo são alimentados exclusivamente com leite materno por pelo menos quatro meses de vida, e comumente as práticas de alimentação complementar são inoportunas, inapropriadas e inseguras. Esse quadro poderia ser revertido com a melhoria do estado nutricional das mulheres em idade fértil (durante a gravidez, especialmente), da amamentação exclusiva durante os seis primeiros meses de vida e de uma alimentação complementar sadia e apropriada até os dois anos de idade ou mais. ${ }^{6}$

Autilização de alimentos artificiais, comparada ao leite materno, pode levar a maior ingestão alimentar, predispondo, assim, à obesidade, à hipercolesterolemia no adulto e ao desencadeamento de doenças ateroscleróticas. Crianças que são alimentadas com leite materno, além de apresentar menores níveis de colesterol na vida adulta, têm menos riscos de doenças cardiovasculares, pois tal suprimento pode programar o metabolismo de gordura. ${ }^{1}$

Sendo assim, é notável a necessidade de a criança ter uma alimentação satisfatória desde o nascimento, pois, caso a mãe não consiga alimentar o seu filho com leite materno exclusivamente até os seis meses de vida e, posteriormente, com alimentação complementar e o leite materno até os dois anos de idade, pode haver maior suscetibilidade às doenças prevalentes na infância.

Sendo assim, este estudo tem por objetivos identificar os principais agravos de saúde em crianças de zero a seis meses de vida e verificar a relação entre os agravos de saúde e as práticas alimentares mais frequentes

\section{METÓDO}

Realizou-se estudo descritivo com abordagem quantitativa, com amostra formada por 36 crianças entre zero e seis meses de idade, acompanhadas pela Enfermagem na Unidade de Cuidado de Enfermagem do Centro de Desenvolvimento Familiar (CEDEFAM) da Universidade Federal do Ceará.

A seleção das crianças ocorreu de forma aleatória de acordo com a demanda do serviço, quando a mãe se encontrava com o seu filho de zero a dois meses de vida na consulta de Enfermagem no CEDEFAM, adotando os seguintes critérios: crianças que tinham entre zero e dois meses de vida e mães das crianças que aceitassem participar do acompanhamento da criança pelo menos até o sexto mês de vida, em aleitamento materno ou não.

Foram adotadas, para este estudo, as categorias de aleitamento materno propostas pela Organização Mundial de Saúde: aleitamento materno exclusivo (AME) - quando a criança recebe somente o leite materno, diretamente da mama ou extraído, e nenhum outro líquido ou sólido, com exceção de gotas ouxaropes de vitaminas, minerais e/ou medicamentos; aleitamento materno predominante (AMP) - quando o lactente recebe, além do leite materno, água ou bebidas à base de água, como sucos de frutas e chás; e aleitamento materno (AM) - quando a criança recebe leite materno, diretamente do seio ou extraído, independentemente de estar recebendo qualquer alimento ou líquido, incluindo leite não humano. ${ }^{7}$ Ressalta-se que o termo desmame foi utilizado relativamente à interrupção total da amamentação. ${ }^{8}$

Para coleta dos dados, utilizou-se formulário que constou de dados referentes às condições socioeconômicas das mães, indicadores relativos à criança, tipo de alimentação e agravos à saúde ocorridas durante o mês. Os agravos à saúde das crianças foram classificados em doenças respiratórias [infecção das vias aéreas superiores (IVAS) ou infecção das vias aéreas inferiores (IVAI)], dermatológicas e gastrointestinais. Os sinais/sintomas das IVAS que foram investigados foram: coriza, obstrução nasal, otorreia e tosse seca. Os sinais/sintomas das IVAl observados foram: tosse produtiva, ausculta pulmonar com presença de roncos/ sibilos/estertores, taquipneia ou dispneia e febre.

Para as crianças acometidas por doenças dermatológicas, foram abordados os seguintes sinais: eritema por contato na face, 
tórax, abdome, glúteo ou raiz das coxas; placas brancas e eritema na língua, mucosa vaginal ou região das dobras; pápulas eritematocrostosas, descamativas e pruriginosas nos pés, mãos, cintura ou nádegas; lesões maculosas hipocrômicas descamativas; lesões urticadas em razão da hipersensibilidade a toxinas de insetos; erupção vesiculopustulosa/crostas purulentas com odor fétido; pequenas vesículas claras em virtude da obstrução dos dutos sudoríparos; lesões eritematodescamativas no couro cabeludo, face e grandes dobras. Crianças com problemas gastrointestinais foram classificadas quanto a diarreia e constipação intestinal.

O estudo foi aprovado pelo Comitê de Ética da Universidade Federal do Ceará - UFC. Após aprovação, as mães aceitaram participar mediante o esclarecimento do seu objetivo, assinando um termo de consentimento pós-informação, conforme a Resolução n 196/96 do Conselho Nacional de Saúde, sendo garantido o anonimato. Foram utilizados cognomes para identificação, garantindo-se o sigilo e a liberdade de desistir da pesquisa, em qualquer momento. Foi assegurado também às participantes o livre acesso ao conteúdo da pesquisa. ${ }^{9}$

Os dados foram tabulados em planilha eletrônica (Excel) e processados no Software Package for the Social Sciences (SPSS 13.0), fixando para todos os testes estatísticos o nível de significância de $p<0,05$. Vale ressaltar que os resultados foram discutidose analisados de acordo com a literatura.

\section{RESULTADO E DISCUSSÃO}

De acordo com a Tabela 1, pode-se verificar que não zero a seis meses acompanhadas (idade: $p=0,508$; número de filhos: $p=0,703$; escolaridade: não houve comparação estatística, pois "agravos à saúde" é uma constante; ocupação: p=0,169; renda: $p=0,492$ ). Isso ocorreu porque a amostra era homogênea, ou seja, pertencente ao mesmo nível socioeconômico e ao mesmo nível cultural.

Tabela 1. Distribuição do número de crianças que apresentaram agravos à saúde segundo os dados sociodemográficos das mães. (Fortaleza, CE, 2006-2007).

\begin{tabular}{|c|c|c|c|c|c|}
\hline \multirow{3}{*}{ Dados sociodemográficos } & \multicolumn{4}{|c|}{ Agravos à saúde } & \multirow[t]{3}{*}{$\mathbf{p}^{*}$} \\
\hline & \multicolumn{2}{|c|}{ Sim } & \multicolumn{2}{|c|}{ Não } & \\
\hline & $\mathrm{N}$ & $\%$ & $\mathrm{~N}$ & $\%$ & \\
\hline \multicolumn{6}{|l|}{ Idade (ano) $(\mathrm{n}=33)$} \\
\hline Menor que 20 & 10 & 30,3 & & & \\
\hline $21-30$ & 16 & 48,5 & 1 & 3,0 & 0,508 \\
\hline $31-36$ & 6 & 18,2 & & & \\
\hline \multicolumn{6}{|l|}{$N^{o}$ de filhos $(\mathrm{n}=35)$} \\
\hline 1 & 12 & 34,2 & 1 & 2,9 & 0,703 \\
\hline $2-4$ & 21 & 60 & 1 & 2,9 & \\
\hline \multicolumn{6}{|l|}{ Escolaridade $(\mathrm{n}=22)$} \\
\hline $\begin{array}{l}\text { Ensino fundamental incompleto (menor ou } \\
\text { equivalente) }\end{array}$ & 11 & 50 & & & \\
\hline $\begin{array}{l}\text { Ensino fundamental completo (maior ou } \\
\text { equivalente) }\end{array}$ & 11 & 50 & & & \\
\hline \multicolumn{6}{|l|}{ Ocupação $(\mathrm{n}=33)$} \\
\hline Dona de casa & 28 & 84,9 & 1 & 3,0 & 0,169 \\
\hline Trabalham fora de casa & 3 & 9,1 & 1 & 3,0 & \\
\hline \multicolumn{6}{|l|}{ Renda familiar $(S M)(\mathrm{n}=28)$} \\
\hline$<1$ & 3 & 10,7 & & & 0,492 \\
\hline $1-4$ & 23 & 82,2 & 2 & 7,1 & \\
\hline
\end{tabular}

Legenda: SM - Salário Mínimo = R\$350,00; *Calculado pelo teste Razão de Verossimilhança. 
Vale salientar, porém, que, das 35 crianças acompanhadas, $33(91,7 \%)$ apresentavam problemas de saúde, sendo que $16(48,5 \%)$ eram provenientes de mães na faixa etária entre 21 e 30 anos, que tinham entre dois e quatro filhos ( $21 \%$ a $60 \%$ ) e eram donas de casa, sua principal ocupação. (28\% a 84,9\%). A renda familiar entre um e quatro salários mínimos foi predominante $(23 \%$ a $82,2 \%$ ) e surpreendentemente foi o estrato social em que houve o maior número de crianças com problemas de saúde (sendo os problemas respiratórios os mais frequentes, não somente nesta variável, mas também em todas as outras).

Mesmo observando que a maioria das mães era de dona de casa, $28(84,9 \%)$ crianças tiveram algum tipo de agravo à saúde, ou seja, a presença das mães durante os primeiros meses de vida da criança não minimizou a ocorrência de afecções nesta faixa etária.

Este estudo apresentou dados significativos segundo os quais muitas mães, mesmo estando em casa, deixaram precocemente de amamentar exclusivamente seus bebês. Surgem, porém, questionamentos, pois sabe-se que fatores intrínsecos a essas mães, como pouca experiência, baixa escolaridade e renda familiar, além de crenças e tradições antigas, influenciam negativamente na prestação dos cuidados à saúde da criança.

Para o desenvolvimento da saúde da criança, no entanto, é essencial a organização do ambiente físico e do entorno da criança, por meio de cuidados consequentes de fatores como cultura, nível socioeconômico, estrutura familiar e características da própria criança. Assim, esta contará com um potencial de resistência ante as adversidades físicas ou psicológicas. $^{10}$

Das 36 crianças acompanhadas no estudo, 33 apresentaram agravos à saúde (problemas respiratórios, dermatológicos e gastrointestinais), com uma ou mais intercorrências, ou seja, uma criança pode ter apresentado mais de uma vez o mesmo tipo de agravo à sua saúde ou haver adquirido outro agravo (com mais de um sinal/sintoma característico), em encontros diferentes ou no mesmo encontro. Sendo assim, nesta tabela, foi considerado como ocorrência cada sinal/sintoma do tipo de problema de saúde que a criança apresentara no encontro.
Em relação às afecções respiratórias, os sinais/ sintomas mais frequentes estavam relacionados às infecções das vias aéreas superiores, como, por exemplo, coriza (32 ocorrências - 17,3\%) que afetou $19(14,5 \%)$ crianças. Já com relação às infecções das vias aéreas inferiores, a tosse produtiva teve 16 $(8,7 \%)$ ocorrências, manifestando-se em $10(7,7 \%)$ lactentes (Tabela 2).

As infecções respiratórias são as causas mais comuns de doenças nas crianças. Lactentes de menos de três meses de vida apresentam menor taxa de infecção por possuírem os anticorpos maternos como proteção. Essa taxa aumenta quando a criança se encontra entre o terceiro e o sexto mês de vida, período em que há diminuição dos anticorpos maternos e quando se inicia a produção dos anticorpos do lactente. ${ }^{11}$

No que diz respeito ao problema dermatológico, pôde-se constatar $81(43,8 \%)$ episódios, ou seja, sinais e sintomas característicos, em 58 (44,2\%) crianças, sendo a presença de eritema por contato na face, tórax, abdome, glúteo ou raiz das coxas o sinal mais frequente, identificado $21(11,3 \%)$ vezes em $17(13 \%)$ crianças. Vale destacar também a presença de lesões urticadas em razão da hipersensibilidade a toxinas de insetos, 0 segundo sinal mais frequente, identificadas $15(8,1 \%)$ vezes em oito $(6,1 \%)$ crianças.

A dermatite de contato, que sugestivamente atingiu a maioria das crianças do estudo, é uma reação inflamatória da pele às diversas substâncias (naturais ou sintéticas) que causam irritação. Varia desde um eritema leve até 0 aparecimento de bolhas sobre uma base eritematosa e edemaciada. Agentes comuns que produzem respostas dermatológicas e são usados comumente em lactentes são perfumes, sabonetes e fraldas. $^{12}$ 
Tabela 2.Distribuição do número de ocorrência dos sinais/sintomas dos problemas de saúde das crianças. (Fortaleza, CE, 2006-2007).

\begin{tabular}{|c|c|c|c|c|}
\hline Problemas de Saúde & $\begin{array}{c}\mathbf{N}^{\mathbf{0}} \text { de } \\
\text { Ocorrência } \\
\text { S } \\
\end{array}$ & $\%$ & $\begin{array}{c}\mathrm{N}^{0} \text { de } \\
\text { Criança } \\
\text { s } \\
\end{array}$ & $\%$ \\
\hline \multicolumn{5}{|l|}{ Respiratório } \\
\hline \multicolumn{5}{|l|}{ IVAS } \\
\hline Coriza & 32 & 17,3 & 19 & 14,5 \\
\hline Obstrução nasal & 8 & 4,3 & 6 & 4,6 \\
\hline Otorreia & 2 & 1,0 & 2 & 1,5 \\
\hline Tosse seca & 9 & 4,9 & 5 & 3,8 \\
\hline \multicolumn{5}{|l|}{ IVAI } \\
\hline Ausculta pulmonar alterada & 10 & 5,4 & 7 & 5,3 \\
\hline Febre & 8 & 4,3 & 8 & 6,1 \\
\hline Taquipneia ou dispneia & 10 & 5,4 & 8 & 6,1 \\
\hline Tosse produtiva & 16 & 8,7 & 10 & 7,7 \\
\hline \multicolumn{5}{|l|}{ Problemas Dermatológicos } \\
\hline Eritema por contato & 21 & 11,3 & 17 & 13,0 \\
\hline Placas brancas e eritema & 13 & 7,0 & 9 & 6,9 \\
\hline $\begin{array}{l}\text { Pápulas eritematocrostosas, } \\
\text { descamativas e pruriginosas }\end{array}$ & 9 & 4,9 & 4 & 3,0 \\
\hline Lesões maculosas hipocrômicas & 4 & 2,2 & 3 & 2,3 \\
\hline Lesões urticadas & 15 & 8,1 & 8 & 6,1 \\
\hline $\begin{array}{l}\text { Erupção vesiculopustulosa/crostas } \\
\text { purulentas com odor fétido }\end{array}$ & 2 & 1,1 & 2 & 1,5 \\
\hline Pequenas vesículas claras & 12 & 6,5 & 11 & 8,4 \\
\hline Lesões eritematodescamativas & 5 & 2,7 & 4 & 3,0 \\
\hline \multicolumn{5}{|l|}{ Problemas Gastrointestinais } \\
\hline Constipação intestinal & 5 & 2,7 & 4 & 3,05 \\
\hline Diarreia & 4 & 2,2 & 4 & 3,05 \\
\hline TOTAL & 185 & 100,0 & 131 & 100,0 \\
\hline
\end{tabular}

Os problemas gastrointestinais foram divididos em constipação intestinal, que teve o maior número de ocorrências $(n=5 ; 2,7 \%)$ e diarreia $(n=4 ; 2,2 \%)$. Além de um agravo à saúde, a diarreia é também um sintoma resultante de algum processo inflamatório de origem infecciosa (infecções das vias aéreas, por exemplo) e pelo uso de antibioticoterapia. A diarreia, no entanto, é um sintoma identificado, dependendo do número de evacuações e da mudança na consistência das fezes, com a tendência de estas ficarem esverdeadas, com muco ou sangue. Medidas como incentivo ao aleitamento materno, troca de fraldas, para manter a pele limpa e seca, e avaliação dos sinais vitais são imprescindíveis para observar a melhora de tal problema. ${ }^{11}$

A família, ao pensar que a diarreia é parte do desenvolvimento normal da criança, reduz a importância de tal agravo, o que mostra como condições de risco para estas crianças as práticas de saúde e as crenças a elas intrínsecas. Cada família, de acordo com as experiências vividas, avalia o teor de gravidade da doença, o que explica o fato de ela buscar ajuda mais rapidamente ou não a um serviço de saúde. ${ }^{13}$

Pode-se observar na Tabela 3 que, quanto ao tipo de alimentação das crianças entre zero e seis meses de vida, acompanhadas independentemente do encontro, a maioria dos lactentes estava em aleitamento materno exclusivo (considerando-se a faixa etária menor do que três meses de idade).

Vale ressaltar que, das 13 (9,7\%) crianças entre zero e um mês de vida, 12 (9\%) estavam em aleitamento materno exclusivo e somente uma $(0,7 \%)$, em aleitamento materno. Com relação às $24(18 \%)$ crianças que se encontravam na 
faixa etária entre um e dois meses de vida, um número significativo estava em aleitamento materno exclusivo $(n=15$; $11,2 \%)$, mas é oportuno salientar que quatro (3\%) crianças estavam em aleitamento materno predominante e duas (1,5\%) já se encontravam desmamadas. Das $31(23,1 \%)$ que estavam na faixa etária entre dois e três meses de vida, 18 (13,4\%) ainda estavam em aleitamento materno exclusivo, porém já havia sete $(5,2 \%)$ crianças em aleitamento materno e três (2,3\%) já haviam sido desmamadas.

Ante este contexto, convém referir que a educação alimentar da criança começa nos primeiros meses de vida, contribuindo para providenciar os alicerces dos costumes alimentares. A forma como a criança se alimenta é determinada pela interação do lactente com o alimento, pelo seu desenvolvimento anatomofisiológico, pelos fatores psicológicos, socioeconômicos, culturais e emocionais e pela interação da criança com a própria mãe ou a pessoa mais ligada à sua alimentação. $^{14}$
Conforme pesquisa do Datasus, $79,4 \%$ das crianças entre zero e 30 dias de vida e $52 \%$ entre 61 e 90 dias, nascidas na cidade de Fortaleza-CE, encontram-se em aleitamento materno exclusivo, o que reafirma os dados obtidos com o este estudo. ${ }^{15}$

Sabe-se que, das crianças entre três e quatro meses de vida, já se percebe uma inversão do tipo de alimentação. Das $32(24 \%)$ crianças, $14(10,5 \%)$ já estavam em aleitamento materno, enquanto dez $(7,4 \%)$ estavam sob aleitamento materno exclusivo. Apesar de as mães serem donas de casa e sem trabalho fixo, pode-se verificar um número significativo de crianças em desmame ( $6 \%$ a $4,5 \%$ ), o que não condiz com a ocupação das mães, as quais poderiam estar mais disponíveis para amamentar seus filhos em casa. Já nos lactentes entre quatro e seis meses de vida, observa-se uma redução importante do aleitamento materno exclusivo, sendo notório o fato de que muitas crianças já estavam desmamadas, representando, assim, um número considerável (12\% a 8,9\%).

Tabela 3. Distribuição do número de crianças segundo o tipo de alimentação e a faixa etária. Fortaleza, CE, 2006-2007

\begin{tabular}{lcccccccccc}
\hline \multicolumn{10}{c}{ Tipo de Alimentação } \\
\hline \multicolumn{1}{c}{ AME } & \multicolumn{1}{c}{ AMP } & \multicolumn{2}{c}{ AM } & \multicolumn{2}{c}{ DESMAME } & \multicolumn{2}{c}{ TOTAL } \\
\hline Idade & $\mathrm{N}$ & $\%$ & $\mathrm{~N}$ & $\%$ & $\mathrm{~N}$ & $\%$ & $\mathrm{~N}$ & $\%$ & $\mathrm{~N}$ & $\%$ \\
$0 \dashv 1$ & 12 & 9,0 & & & 1 & 0,7 & & & 13 & 9,7 \\
$1 \dashv 2$ & 15 & 11,2 & 4 & 3,0 & 3 & 2,3 & 2 & 1,5 & 24 & 18,0 \\
$2 \dashv 3$ & 18 & 13,4 & 3 & 2,2 & 7 & 5,2 & 3 & 2,3 & 31 & 23,1 \\
$3 \dashv 4$ & 10 & 7,4 & 2 & 1,6 & 14 & 10,5 & 6 & 4,5 & 32 & 24,0 \\
$4 \dashv 5$ & 6 & 4,5 & 3 & 2,2 & 7 & 5,2 & 7 & 5,2 & 23 & 17,1 \\
$5 \dashv 6$ & 1 & 0,7 & 3 & 2,2 & 2 & 1,5 & 5 & 3,7 & 11 & 8,1 \\
\hline TOTAL & 62 & 46,2 & 15 & 11,2 & 34 & 25,4 & 23 & 17,2 & 134 & 100,0 \\
\hline
\end{tabular}

Legenda: AME = Aleitamento materno exclusivo; AMP $=$ Aleitamento materno predominante; $\mathrm{AM}=$ Aleitamento materno;DESMAME $=$ Crianças desmamadas.

0 ato de amamentar é uma antiga prática com reconhecimento quanto aos diversos benefícios nutricionais, imunológicos, cognitivos, econômicos e sociais, estes percebidos quando a amamentação é feita até os dois anos de idade e exclusivamente até os seis meses de vida da criança. ${ }^{8}$

Segundo a pesquisa do Datasus, $86,6 \%$ das crianças entre 91 e 120 dias de vida, nascidas na cidade de FortalezaCE, estão em aleitamento materno, mostrando o quão precocemente as crianças estão sendo introduzidas na alimentação complementar, permitindo que o leite materno, não sendo a única fonte de alimentação, exerça menos eficazmente seu papel de proteção à criança. ${ }^{15}$

A introdução precoce de alimentos suplementares, como água e chás, e complementares, como frutas e papas salgadas, pode resultar na redução do tempo de amamentação. Tal prática pode ser explicada em parte por fatores culturais e em razão da falta de conhecimento de alguns profissionais da área da saúde acerca dos benefícios do aleitamento materno exclusivo durante os seis primeiros meses de vida. ${ }^{8,16}$

A Tabela 4 demonstrou que, em relação às ocorrências de agravos à saúde, das crianças que estavam em aleitamento materno exclusivo, houve $75(40,5 \%)$ casos, sendo 33 $(17,8 \%)$ problemas respiratórios, 38 (20,5\%) dermatológicos e $4(2,2 \%)$ gastrointestinais.

0 fato de amamentar exclusivamente 0 bebê confere intensa proteção contra óbito por diarreia. 0 aleitamento materno predominante ou o desmame estão associados ao 
risco 3,9 vezes maior de morte por infecções respiratórias e diarreia. ${ }^{14}$

Mesmo tendo observado um número significativo de crianças com problemas de saúde, em aleitamento materno exclusivo, é oportuno salientar que outros aspectos podem influenciar o aparecimento dessas ocorrências, tais como: condições ambientais, vínculo mãe-filho, questões familiares e emocionais. Isto se comprova com a verificação, neste estudo, da não existência de agravos à saúde de 21 (56,8\%) crianças com este tipo de alimentação.

Tabela 4. Distribuição do número de ocorrências dos principais agravos à saúde das crianças segundo o tipo de alimentação. (Fortaleza, CE, 2006-2007).

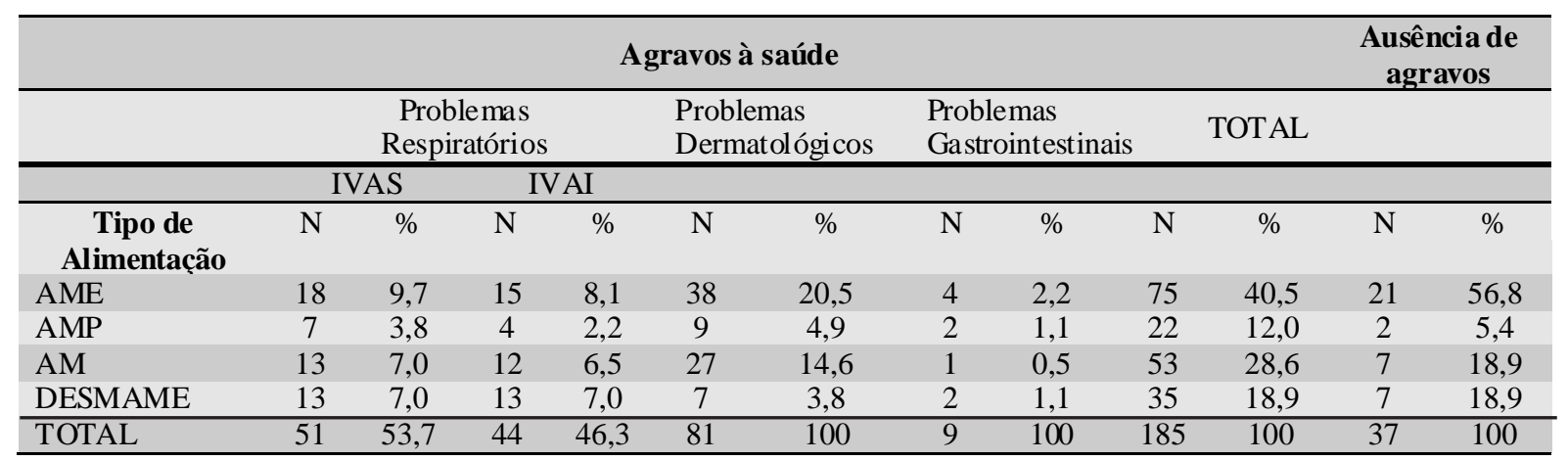

Legenda: AME = Aleitamento materno exclusivo; $\mathrm{AMP}=$ Aleitamento materno predominante; $\mathrm{AM}=$ Aleitamento materno.

Dentre as 22 (12\%) ocorrências de agravos à saúde de crianças em aleitamento materno predominante, houve 11 (6\%) relacionadas aos problemas respiratórios (sete ocorrências de IVAS - $3,8 \%)$ e nove $(4,9 \%)$ aos problemas dermatológicos. Vale ressaltar que em somente duas $(5,4 \%)$ crianças com este tipo de alimentação não ocorreram agravos à sua saúde.

Quanto às 53 (28,6\%) ocorrências de afecções à saúde em crianças em aleitamento materno, houve $27(14,6 \%)$ relacionadas aos problemas dermatológicos e 25 (13,5\%) vinculadas aos problemas respiratórios, sendo $13(7 \%)$ ocorrências de IVAS. É importante lembrar que em somente sete $(18,9 \%)$ crianças com este tipo de alimentação não houve presença de agravos à saúde.

A introdução precoce de água, sucos e chá é desnecessária para a hidratação do bebê e pode elevar o risco de morbimortalidade por infecções, além de não promover ganho ponderal e fazer reduzir a absorção de ferro e zinco. ${ }^{8}$ Além disso, a introdução de alimentos complementares pode aumentar o risco de alergia alimentar e a ocorrência de doenças crônicodegenerativas na idade adulta. ${ }^{14}$

Com relação às crianças desmamadas, houve 35 (18,9\%) ocorrências de problemas de saúde; de maior destaque foram 26 (14\%) ocorrências de problemas respiratórios (sendo o aparecimento das IVAS e IVAI em igual proporção - 7\%), enquanto houve sete $(3,8 \%)$ ocorrências de problemas dermatológicos.

0 estudo mostra que realmente a criança desmamada fica mais suscetível às afecções, principalmente as respiratórias, que atingem o trato inferior do aparelho respiratório (mais graves), quando comparada às crianças em aleitamento materno exclusivo, aleitamento materno predominante e aleitamento materno.

Crianças precocemente desmamadas têm risco relativo de óbito (em razão das doenças respiratórias agudas, da diarreia e outras patologias infecciosas) mais de 20 vezes mais elevado, o que indica o fator de proteção que a amamentação exerce nas crianças. ${ }^{17}$

0 ato de amamentar, além de um processo fisiológico, natural, constitui a melhor forma de alimentar e proteger a criança. É útil lembrar, porém, que a amamentação é algo que se aprende, precisando de tempo, prática e paciência para tornar esse ato uma experiência confortável e bem-sucedida. Por exemplo, algumas mães têm medo de cuidar do filho e outras têm dificuldades para amamentar, o que pode tumultuar o relacionamento mãe-filho. Apesar disso, felizmente, em sua maioria, os problemas surgidos durante a amamentação podem ser solucionados com alguns ajustes muito simples. 


\section{Práticas alimentares e intercorrências clínicas}

\section{CONCLUSÃO}

Ante o exposto, pode-se concluir que muitas das mães, mesmo estando em casa, deixaram precocemente de amamentar exclusivamente seus bebês. Há, no entanto, fatores intrínsecos a essas mães que influenciam na prestação dos cuidados à saúde da criança.

A figura materna, principal cuidadora do lactente, é um dos principais elos para o crescimento e o desenvolvimento satisfatório dele, promovendo, juntamente com a família, os principais vínculos para um cuidado com qualidade, tanto nos âmbitos físico e afetivo como social.

As crianças, principalmente as menores de seis meses de idade, fazem parte de um grupo mais vulnerável, pelo fato de ainda estarem biologicamente pouco amadurecidas. Por conta disso, é possível que o adoecimento, dependendo do agente infeccioso, cause o agravamento do quadro de saúde da criança, podendo levá-la à hospitalização. 0 leite materno, tendo incontestáveis vantagens, pode reduzir a morbimortalidade infantil, principalmente por doenças alérgicas e respiratórias e por diarreia.

Para que haja aumento nos índices de aleitamento materno exclusivo, é necessário, tanto da parte do governo como dos profissionais de saúde, uma visão do aleitamento materno como um valor social, focando não somente a mulher, mas também a sociedade como um todo. Deve-se dar maior atenção à prevenção de situações que propiciam o nascimento de bebês de baixo peso, além de medidas como melhoria no atendimento pré-natal, por meio da Educação em Saúde.

Vê-se, portanto, a real necessidade da criação de outras medidas governamentais com o intuito de levantar projetos que intensifiquem cada vez mais a promoção do aleitamento materno, como ações adequadas de incentivo de vendas de alimentos para bebês (com medidas corretivas apropriadas depois dos seis meses de idade) e de apoio individual, familiar e social às mães, principalmente àquelas que trabalham fora de casa durante o período de lactação. Além disso, é importante o desenvolvimento de atividades educacionais por enfermeiros (e também por outros profissionais da área da saúde) que visem ao prolongamento da duração do aleitamento materno exclusivo até os seis meses de idade, para reduzir, assim, a morbimortalidade infantil.

Outra ação indispensável é a capacitação dos enfermeiros responsáveis pela puericultura no acompanhamento das mães e bebês incluídos nesse grupo, no sentido de incentivar e apoiar o aleitamento materno exclusivo até, no mínimo, seis meses de vida e identificar precocemente os principais agravos à saúde da criança.

\section{REFERÊNCIAS}

1. Souza SL, Castro RM, Nogueira MI. Comportamento alimentar neonatal. Rev Bras Saude Matern Infant 2003; 3(3): 241-46.

2. Silva MBC, Moura MEB, Silva AO. Desmame precoce: representaç̃̃es sociais de mães. Rev Eletr Enferm [on-line]. 2007; 9(1): 31-50. Available from: http://www.fen.ufg.br/revista/v9/n1/v9n1a03.htm

3. Mascarenhas MLW, Albernaz EP, Silva MB, Silveira RB. Prevalência de aleitamento materno exclusivo nos três primeiros meses de vida e seus determinantes no Sul do Brasil. J Pediatr 2006; 82 (4): 289-94.

4. Nejar FF, Segall-Corrêa AM, Rea MF, Vianna RPT, Panigassi G. Padrões de aleitamento materno e adequação energética. Cad Saude Publica 2004; 20(1): 64-71.

5. Venancio SI, Escuder MML, Kitoko P, Rea MF, Monteiro CA. Frequência e determinantes do aleitamento materno em municípios do Estado de São Paulo. Rev. Saude Publica. 2002; 36(3): 313-18.

6. Resolução AMS n 55.25/2002. Nutrição do lactente e da criança pequena. [citado 24 mar 2007] Disponível em: http://www.ibfan.org/ portuguese/resource/who/whares5525-po.html.

7. Organização Panamericana da Saúde-OPS. Atenção integrada às doenças prevalentes na infância (AIDPI): manual de capacitação em atenção primária. Fortaleza(CE): Escola de Saúde Pública; 2005. 117p.

8. Chaves RG, Lamounier JÁ, César CC. Fatores associados com a duração do aleitamento materno. J Pediatr 2007; 83(3): 241-46.

9. Ministério da Saúde (BR).Conselho Nacional de Saúde de Ética em Pesquisa. Resolução 196/96 sobre pesquisa envolvendo seres humanos. Brasília (DF); 1996.

10. Martins MFD, Costa JSD, Saforcada ET, Cunha MDC. Qualidade do ambiente e fatores associados: um estudo em crianças de Pelotas, Rio Grande do Sul, Brasil. Cad Saude Publica. 2004; 20(3): 710-18.

11. Collet N, Oliveira BRG. Enfermagem pediátrica. Goiânia(G0): AB; 2002. 185p.

12. Whaley LF, Wong DL. Enfermagem pediátrica: elementos essenciais à intervenção efetiva. $5^{a}$. ed. Rio de Janeiro(RJ): Guanabara Koogan; 1999. 1118p.

13. Feliciano KVO, Kovacs MH. As mães são estimuladas a valorizar os sinais de risco da diarreia. J Pediatr 1998; 74(2): 135-42.

14. Vieira GO, Silva LR, Vieira TO, Almeida JAG, Cabral VA. Hábitos alimentares de crianças menores de 1 ano amamentadas e nãoamamentadas. J Pediatr 2004; 80(5): 411-16.

15. Ministério da Saúde (BR). DATASUS. Indicadores Demográficos e Socioeconômicos - 2005 [citado 23. maio 2007]

Disponível em <http://tabnet.datasus.gov.br/cgi/idb2005/ matriz.htm>. 
16. Osorio CM, Queiroz ABA. Representações sociais de mulheres sobre a amamentação: teste de associação livre de ideias acerca da interrupção precoce do aleitamento materno exclusivo. Esc Anna Nery Rev Enferm 2007; 11(2): 261-67.

17. Bittencourt $\bigsqcup$, Oliveira JS, Figueiroa JN, Batista Filho M. Aleitamento materno no estado de Pernambuco: prevalência e possível papel das ações de saúde. Rev Bras Saude Matern Infant 2005; 5(4): 439-48. 